\title{
Resultats A Court Terme De La Trabeculoplastie Selective Au Laser Chez Les Patients Togolais
}

\author{
Dzidzinyo Kossi \\ Djagnikpo Akouete \\ Service d'ophtalmologie, CHU Sylvanus Olympio, \\ Université de Lomé, Lomé, Togo \\ Ayena Koffi Didier \\ Service d'ophtalmologie, Hôpital Secondaire de Bè, \\ Université de Lomé, Togo \\ Vonor Kokou \\ Service d'ophtalmologie, CHU Sylvanus Olympio, \\ Université de Lomé, Lomé, Togo

\section{Maneh, Nidain}

Service d'ophtalmologie, CHU Campus, Université de Lomé, Lomé, Togo

Amedome Kokou Messan

Santos Mawuli

Balo Komi

Service d'ophtalmologie, CHU Sylvanus Olympio,

Université de Lomé, Lomé, Togo

Doi: 10.19044/esj.2017.v13n30p271 URL:http://dx.doi.org/10.19044/esj.2017.v13n30p271

\begin{abstract}
Aim: To check the short-term tonometric results of SLT in the treatment of primary glaucoma at the open angle and in charge of ocular hypertonias in Togolese people. Methods: A retrospective study was carried out in an ophthalmology center. The first 130 eyes of 72 patients benefited from the SLT laser procedure. The tonometric controls work object focus on follow-up at 1, 3, and 6 months post laser treatment. Results: 130 eyes of 72 patients were collected. The average age of the patients was 49.74 years $( \pm$ $17.45)$ and the ages vary between 10 and 85 years. The average IOP of the laser before the laser (J0) was $(24.99 \pm 8.41) \mathrm{mm} \mathrm{Hg}$. The mean IOP at the post-laser control at 1 month was $(18.79 \pm 3.73) \mathrm{mm} \mathrm{Hg}$. The average IOP for the post-laser control at 3 months was $(18.44 \pm 3.81) \mathrm{mm} \mathrm{Hg}$. The mean IOP at the post-laser control at 6 months was $(18.13 \pm 3.63) \mathrm{mm} \mathrm{Hg}$. The percentage reduction in intraocular pressure compared to IOP was pretreated from $20.2 \%$ to 1 month; $22.1 \%$ at 3 months; and $23.3 \%$ at 6 months. In 1
\end{abstract}


month, $49.2 \%$ of the eyes we treated showed a reduction in IOP of less than $20 \%$ compared to IOP pretreatment. After 3 months and 6 months, it was $55.4 \%$ higher. Also, 52.3\% have a PIO reduction percentage which is greater than or equal to $20 \%$ compared to pre-treatment IOP. Discussion: Selective laser trabeculoplasty, most especially, is interesting in ocular hypertonies. Treatment of over $180^{\circ}$ allows one patient out of two to have a pressure reduction that is greater than or equal to $20 \%$. Conclusion: The SLT presents a significant advantage for our glaucomatous patients.

Keywords: Glaucoma, ocular hypertension, SLT, IOP reduction

\section{Résumé}

But: vérifier résultats tonométriques à court terme de la SLT dans le traitement du glaucome primitif à angle ouvert et dans la prise en charge des hypertonies oculaires chez le patient togolais. Méthodes: étude rétrospective réalisée dans un centre d'ophtalmologie. Les 130 premiers yeux de 72 patients ont bénéficié d'une procédure de laser SLT. Les contrôles tonométriques objet de ce travail portent sur le suivi à 1,3 et 6 mois post traitement laser. Résultats: 130 yeux de 72 patients avaient été colligé. La moyenne d'âge de nos patients était de 49,74 ans $( \pm 17,45)$ et les âges variaient entre 10 et 85 ans. La moyenne de la PIO avant le laser (J0) était de $(24,99 \pm 8,41) \mathrm{mm} \mathrm{Hg}$. La moyenne de la $\mathrm{PIO}$ au contrôle post laser à 1 mois était de $(18,79 \pm 3,73) \mathrm{mm} \mathrm{Hg}$. La moyenne de la PIO au contrôle post laser à 3 mois était de $(18,44 \pm 3,81) \mathrm{mm} \mathrm{Hg} \mathrm{La} \mathrm{moyenne} \mathrm{de} \mathrm{la} \mathrm{PIO} \mathrm{au} \mathrm{contrôle}$ post laser à 6 mois était de $(18,13 \pm 3,63) \mathrm{mm} \mathrm{Hg}$. Le pourcentage de réduction de la pression intra oculaire par rapport à la PIO pré traitement était de $20,2 \%$ à 1 mois.; $22,1 \%$ à 3 mois et $23,3 \%$ à 6 mois. A 1 mois, $49,2 \%$ de nos yeux traités présentaient un pourcentage réduction de la PIO inferieure à $20 \%$ par rapport à la $\mathrm{PIO}$ pré traitement. A 3 mois et 6 mois ils étaient respectivement de $55,4 \%$ et $52,3 \%$ à avoir un pourcentage de réduction de la $\mathrm{PIO}$ supérieure ou égale à $20 \%$ par rapport à la $\mathrm{PIO}$ pré traitement. Discussion: La trabéculoplastie sélective au laser est particulièrement intéressante dans les hypertonies oculaires. Le traitement sur $180^{\circ}$, a permis chez un patient sur deux d'avoir une réduction pressionnelle supérieure ou égale $20 \%$. Conclusions: La SLT présente un atout non négligeable pour nos patients glaucomateux.

Mots-clés: Glaucome, hypertonie oculaire, SLT, réduction PIO

\section{Introduction}

Le glaucome est une neuropathie optique, d'évolution chronique et progressive, accompagnée généralement d'une hyperpression intraoculaire (HPO), caractérisée par une excavation, des altérations périmétriques, et 
évoluant progressivement vers une atrophie optique .Elle est généralement associée à une hypertonie oculaire. C'est la deuxième cause de cécité dans le monde après la cataracte. La cécité glaucomateuse est irréversible. C'est un problème de santé publique surtout dans les pays au Sud du Sahara. Selon Quigley (1996), on dénombrerait environ 66 millions de cas de glaucomes dans le monde, parmi eux près de 35 millions sont des glaucomes primitifs à angle ouvert.

Les études réalisées à Baltimore sur des groupes de population d'origine diverse ont démontré une forte prévalence du GPAO dans les populations Américaines Africaines, les prévalences étant quatre fois plus élevée comparée à celle des Américains blancs.

Plusieurs études ont démontré la gravité et la sévérité du GPAO chez le mélanoderme, certains facteurs de risque s'associent pour expliquer cette gravité ; ce sont le diagnostic tardif, la non connaissance et le manque d'information sur la maladie de la part des patients, certains facteurs héréditaires, la tendance à de très fortes hypertonies (Balo et al., 2006) notamment. De plus les résultats de la chirurgie sont souvent grevés par la fréquence relative de fibroses cicatricielles réduisant l'efficacité post chirurgie. Il faut aussi souligner les difficultés liées à une faible compliance au traitement qui s'explique par le coût élevé de certains médicaments.

La prise en charge du glaucome et des hypertonies oculaires se posent avec acuité en Afrique au Sud du Sahara. L'arsenal thérapeutique est assez varié. Les moyens utilisés dans la prise en charge de l'hypertonie oculaire ou du glaucome comprennent les collyres hypotonisant, la chirurgie filtrante et les moyens physiques lasers. En raison de la chronicité de la maladie le traitement est au long cours .Ce traitement a inévitablement un coût (Azuara-Blanco \& Burr, 2006; Stein et al., 2012). Aussi des études ont démontré que l'adhésion au traitement médical est un défi majeur pour les patients présentant un glaucome primitif à angle ouvert (Tsai, 2006). Les nouvelles molécules telles les analogues des prostaglandines, ont un coût élevé. Dans notre contexte les soins et les médicaments sont à la charge des patients en l'absence d'un système d'assurance maladie.

Le glaucome évoluant à bas bruit, les patients à un stade de début ou modéré ont une relative bonne vision et ont une réticence à accepter la chirurgie filtrante.

Amener un patient à accepter se faire opérer de glaucome relève de la détermination. Dans les glaucomes évolués la chirurgie est souvent proposée mais les résultats fonctionnels ne sont pas toujours satisfaisants. Les patients le perçoivent comme un échec surtout en comparaison avec les cataractes obturantes opérées.

Alors quelle alternative existe- t-il pour résoudre ces problèmes d'observance, de coût, d'adhésion, de persistance et de complications ? Le 
traitement idéal devrait être facile à utiliser, accessible financièrement et pourvoyeur de peu ou pas de complications.

Le traitement laser répondrait il en partie à ces interrogations ?

La trabéculoplastie au laser Argon (ALT) a été introduit par Wise et Witter en 1979. Cette technique a été vulgarisée jusqu'à ce que ne se posent les problèmes tels que : échecs thérapeutiques à long terme, les hypertonies post laser, les uvéites post traitement, les synéchies périphériques (Shingleton et al., 1993). En 1995 Latina et Park ont décrit la trabeculoplastie sélective au laser (SLT) (Latina \& Park, 1995) comme une alternative thérapeutique. Le laser SLT est un laser Q-switched Neodymium : yttrium-aluminium-garnet ( $\mathrm{Nd}$ : yag) en mode pulsé d'une longueur d'onde de $532 \mathrm{~nm}$ dont l'originalité réside dans son caractère sélectif.

Les cliniciens qui ont des difficultés à avoir une bonne visualisation du trabéculum trouvent la SLT plus aisée que l'ALT en raison de la grande taille du spot (Pham, Mansberger \& Brandt, 2008). La SLT agit préférentiellement sur les cellules pigmentées du trabéculum en détruisant la mélanine sans atteinte des faisceaux de collagène ou des cellules adjacentes sans mélanine. La SLT agit avec des spots de 400um et une puissance plus faible $(0,7-1,5 \mathrm{~mJ})$ en raison de son caractère pulsé. Lors de la procédure, l'évacuation de l'humeur aqueuse es $t$ favorisée par un effet mécanique d'étirement des fibres et par des effets biochimiques par modification du métabolisme du collagène (Kramer \& Noecker, 2001).

Il existe de nombreuses études dans la littérature qui ont rapporté l'efficacité de la SLT sur la pression intra oculaire (Stein \& Challa, 2007); En Afrique noire peu d'études font cas des résultats thérapeutiques du laser SLT. On peut cependant noter l'étude de Seck au Sénégal (Seck et al., 2015) en 2014. En Afrique du Nord et au Maghreb, les études d'Abdelrahman en Egypte font exception (Abdelrahman \& Eltanamly, 2012).

Le présent travail présente une première évaluation tonométrique à court terme du traitement par SLT chez des patients Togolais.

\section{Patients et methodes}

Nous avons réalisé une étude rétrospective qui s'est tenue dans un cabinet d'ophtalmologie de Lomé au Togo qui s'est déroulée du 15 juin 2012 au 15 janvier 2013 et concernait les 130 premiers yeux de 72 patients ayant bénéficié d'une procédure de laser SLT.

\section{Critères d'inclusion}

Ont été inclus dans cette étude, les patients qui ont consulté dans la période de l'étude, qui ont été diagnostiqué présentant un glaucome primitif à angle ouvert, ou déjà suivi pour glaucome. Etaient aussi inclus les patients 
présentant une hypertonie oculaire isolée, de même que les patients adressés pour la procédure laser SLT venant d'autres centres ophtalmologiques de la ville.

Avant le traitement, une information éclairée a été donnée aux patients sur la nature de ce type d'intervention. Nous avions reçu de chaque patient un consentement écrit.

N'ont pas été inclus dans cette étude les patients qui ont déjà subi une chirurgie du glaucome.

\section{Nos définitions opérationnelles}

Nous avons défini comme glaucome primitif à angle ouvert toute neuropathie optique avec les caractéristiques classiques de papille glaucomateuse associé à des altérations du champ visuel avec ou sans élévation de la pression intra oculaire. Etait défini comme hypertonie oculaire isolée une pression intra oculaire supérieure à $21 \mathrm{~mm} \mathrm{hg} \mathrm{sans}$ altération du champ visuel ni modification au niveau de la papille optique. Nous avons défini le pourcentage de réduction de la $\mathrm{PIO}$, comme niveau de baisse de la pression pré traitement jusqu'au contrôle post traitement. Cette réduction est exprimée en pourcentage. La réduction de la $\mathrm{PIO}$ est le différentiel de la PIO pré traitement et la PIO de contrôle. Elle est exprimée en millimètre de mercure $(\mathrm{mm} \mathrm{Hg})$.

Tous les patients avaient bénéficié d'un examen ophtalmologique complet et Les variables recueillies étaient : l'âge le sexe, l'acuité visuelle le degré d'ouverture de l'angle irido cornéen, la pigmentation du trabéculum, la pression intra oculaire de base (avant le laser) et la nature du traitement antérieur pour ceux qui bénéficiaient déjà d'un traitement.

\section{La procédure SLT}

Tous les patients traités au laser SLT ont reçu avant la procédure deux gouttes de collyre anesthésique notamment de l'oxybuprocaïne à $1 \%$. Après installation du patient, un verre à gonioscopie de Latina a été posé avec comme interface verre/cornée une solution visqueuse de méthyle cellulose.

Nous avions utilisé le Laser SLT Nd: yag laser, ELLEX SOLO (Ellex médical laser limited, Adelaide, Australie) émettant une longueur d'onde de $532 \mathrm{~nm}$ avec un temps d'exposition de 3 nanosecondes et une taille du spot de 400um.

Le faisceau laser était focalisé sur le trabéculum de façon à bien visualiser la zone à traiter. L'énergie délivrée était comprise entre 0,6 et 0,9 $\mathrm{mJ}$, elle était jugée suffisante dès obtention de bulles dans la chambre antérieure. Le site traité était le trabéculum inferieur sur les $180^{\circ}$ et le nombre d'impacts était de 50. 
Les patients préalablement traités par les analogues prostaglandiniques avaient arrêté ce traitement au moins 30 jours avant SLT. Ce traitement avait été relayé soit par un bêtabloquant, soit par un alpha 2 adrénergique, ou soit par un inhibiteur de l'anhydrase carbonique.

\section{L'évaluation}

A la fin de la séance de laser deux gouttes de collyre brinzolamide étaient instillées dans l'œil traité. Le patient repartait avec une prescription de diclofenac collyre à raison d'une goutte 4 fois par jour dans l'œil traité pendant 72 heures.

Les patients étaient revus, à un mois (M1), trois mois (M3) et 6 mois (M6) post traitement laser et où la pression intra oculaire avait été recontrôlé. La réponse thérapeutique était jugée positive si la réduction de la $\mathrm{PIO}$ à $\mathrm{M} 1$, M3 et M6 était supérieure ou égale à 20\% par rapport à la PIO initiale. Pour les besoins de l'analyse nous avons considéré trois groupes de PIO : les PIO inferieur ou égale à $25 \mathrm{mmHg}$; les $\mathrm{PIO}$ comprises entre 26 et 35 ; et les PIO supérieur ou égale à $36 \mathrm{~mm} \mathrm{Hg}$.

\section{Resultats}

Au total 130 yeux (67 yeux droits et 63 yeux gauches) de 72 patients avaient été enrôlés dans cette étude. Trente quatre patients étaient de sexe masculin soit $47,22 \%$ et 38 patients de sexe féminin. L'âge moyen de nos patients était de 49,74 $\pm 17,46$ ans (Tableau I) avec des extrêmes allant de 10 à $85 \mathrm{ans}$. Il était de 53,73 ans chez les hommes et 46, 08 ans chez les femmes.

Tableau I. Caractéristiques démographiques et cliniques des patients

\begin{tabular}{cc}
\hline Nombre d'yeux/ patients & $130 / 72$ \\
\hline Genre: & $34 / 38$ \\
masculin/féminin & \\
Age, années: & 49,74 \\
Moyenne & 10 à 85 ans \\
Variation & $104 / 26$ \\
Diagnostic & \\
- GPAO/ HTO & \\
PIO initiale: & $24,99 \mathrm{mmHg}$ \\
Moyenne & 15 à $60 \mathrm{mmHg}$ \\
variation &
\end{tabular}

La moyenne de la PIO avant le laser (J0) était de 24,99 $\mathrm{mmHg} \pm 8,41$ $\mathrm{mm} \mathrm{Hg}$. La PIO initiale variait entre 15 et $60 \mathrm{mmHg}$. La moyenne de la PIO au contrôle post laser à 1 mois était de $18,79 \pm 3,73 \mathrm{~mm} \mathrm{Hg}$ soit une moyenne de réduction de la pression intraoculaire de $6,20 \mathrm{mmHg}$ au premier contrôle. La moyenne de la PIO au contrôle post laser à 3 mois était de 18,44 $\pm 3,81 \mathrm{mmHg}$ soit une moyenne de réduction de la $\mathrm{PIO}$ par rapport à la $\mathrm{PIO}$ pré traitement de $6,55 \mathrm{mmHg}$. La moyenne de la PIO au contrôle post laser à 
6 mois était de 18,13 $\pm 3,63 \mathrm{mmHg}$ soit une moyenne de réduction de la PIO par rapport à la PIO pré traitement de $6,86 \mathrm{mmHg}$. La courbe d'évolution de la pression est ainsi exprimée par la Figure 1.

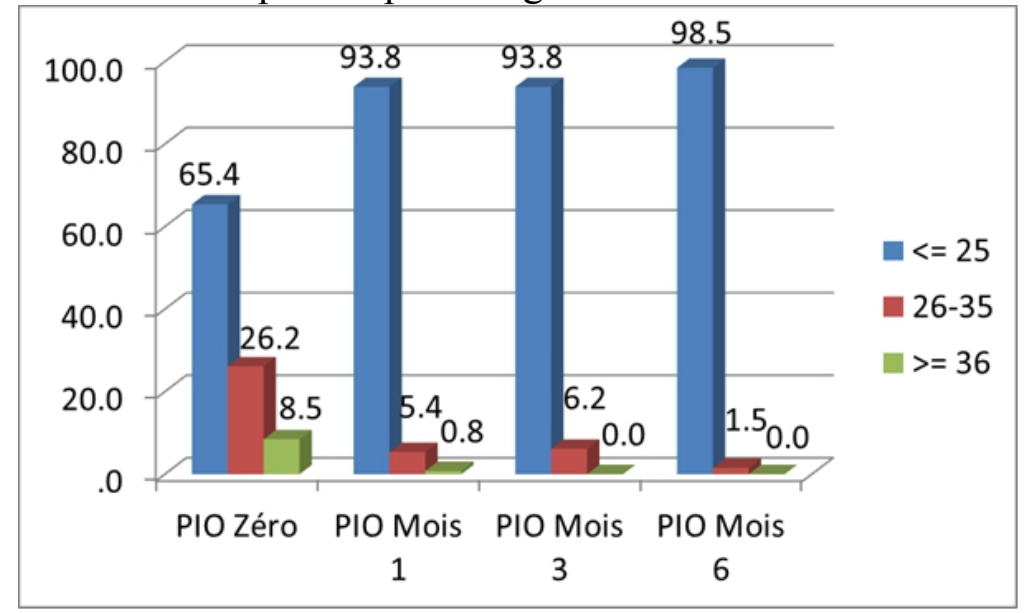

Figure 1. Répartition par strates de la PIO avant et après SLT

Nous avons défini trois groupes de PIO : les PIO inferieur ou égale à $25 \mathrm{mmHg}$; les PIO comprises entre 26 et $35 \mathrm{mmHg}$ et les PIO supérieur ou égale à $36 \mathrm{mmHg}$. Comme le montre la Figure 2, nous objectivons la décroissance des PIO les plus élevés en fonction du temps.

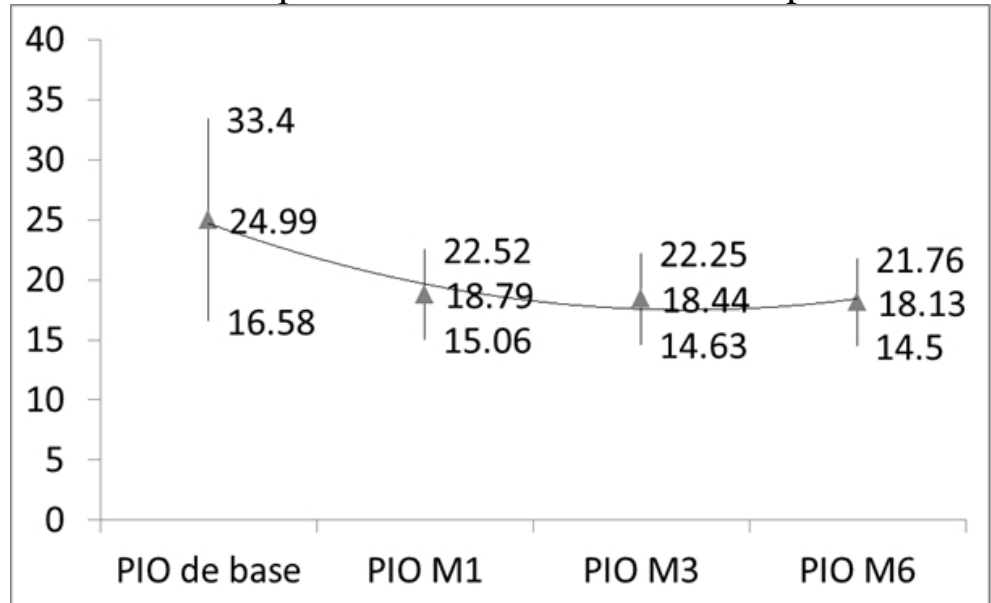

Figure 2. Répartition de la PIO moyenne (en $\mathrm{mmHg}$ ) avant et après traitement SLT

Le pourcentage de réduction de la pression intra oculaire par rapport à la $\mathrm{PIO}$ pré traitement à 1 mois était de $21,1 \%$ à l'œil droit et $19,4 \%$ à l'œil gauche soit $20,2 \%$ pour l'ensemble des yeux traités. Le pourcentage de réduction de la pression intra oculaire par rapport à la $\mathrm{PIO}$ pré traitement à 3 mois était de $22,5 \%$ à l'œil droit et $21,6 \%$ à l'œil gauche soit $22,1 \%$ pour l'ensemble des yeux traités (Figure 3). Le pourcentage de réduction de la 
pression intra oculaire par rapport à la $\mathrm{PIO}$ pré traitement à 6 mois était de $23,8 \%$ à l'œil droit et $22,8 \%$ à 1 'œil gauche, soit $23,3 \%$ à l'ensemble des yeux traités.

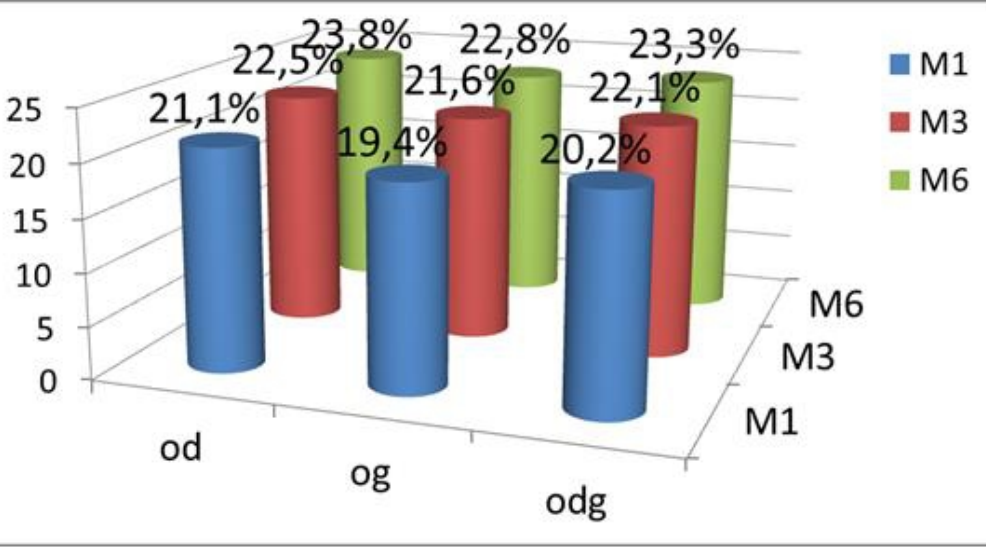

Figure 3. Pourcentage de réduction de la PIO selon l'œil et le contrôle

A 1 mois, 49,2\% de nos yeux traités présentaient un pourcentage réduction de la $\mathrm{PIO}$ inferieure à $20 \%$ par rapport à la $\mathrm{PIO}$ pré traitement (Tableau II). A 3 mois 55,4\% des yeux traités présentaient un pourcentage de réduction de la $\mathrm{PIO}$ supérieure ou égale à $20 \%$ par rapport à la $\mathrm{PIO}$ pré traitement. A 6 mois, $52,3 \%$ des yeux traité avaient un pourcentage de réduction pressionnel maintenue à plus de $20 \%$.

Tableau II. Répartition de la PIO selon le pourcentage de réduction

\begin{tabular}{|c|c|c|c|}
\hline *\% de réduction & $\overline{\mathrm{a}} \neq \mathrm{M} 1$ & à M3 & à M6 \\
\hline$<20 \%$ & $49,2 \%$ & $44,6 \%$ & $47,7 \%$ \\
\hline$>=20 \%$ & $50,8 \%$ & $55,4 \%$ & $52,3 \%$ \\
\hline Total & $100 \%$ & $100 \%$ & $100 \%$ \\
\hline
\end{tabular}

\section{Discussion}

L'âge moyen de notre population d'étude était de 49,74 ans. Seck et al au Sénégal avait retrouvé 58 ans pour une population variant de 37 à 85 ans. Contrairement aux études sur le traitement laser, nous avions enregistré des patients relativement très jeunes. L'âge minimal était de dix ans ; et il s'agissait d'une hypertonie à 32 et $24 \mathrm{~mm}$ hg. Ceci vient souligner une fois encore que le glaucome est précoce et plus sévère chez le sujet mélanoderme. Balo au Togo en 1994 avait trouvé que 66,87\% des patients glaucomateux avaient moins de 45 ans parmi lesquels 7,25\% étaient de la tranche d'âge de 11 à 15 ans.

Tandis que 65 des 69 yeux de l'étude sénégalaise étaient des glaucomes chroniques, la notre retrouve 104 glaucome chronique pour 26 
hypertonies oculaires isolés. La PIO pré traitement dans notre étude était de $24,99 \pm 8,41 \mathrm{~mm}$ hg. Cette PIO de base semble élevée comparé à celle de Shazly (Shazly et al., 2012) et Almeida (Almeida Junior et al., 2011) qui avait retrouvé respectivement une PIO de $23,8 \pm 3,1 \mathrm{~mm}$ hg et $19,2 \pm 2,3 \mathrm{~mm}$ hg. Seck a retrouvé une PIO de base de $18,3 \mathrm{mmHg}$. Cette observation vient une fois encore corroborer les fortes hypertonies dans notre population (Balo et al., 2006).

Plusieurs études ont démontré l'efficacité de la SLT comme une alternative valable et très efficace dans la prise en charge du glaucome primitif à angle ouvert et les hypertonies oculaires (Melamed et al., 2003; Latina et al., 2002; Latina et al.,1996; Damji et al., 1999).

Par quel mécanisme peut-on avoir une réduction de la PIO par traitement laser au niveau du trabéculum ? Il existe deux théories (Murthy \& Latina, 2009) l'expliquant: la théorie mécanique suggère un étirement des fibres lors de la photo dysruption trabéculaire ce qui favorise l'évacuation de l'humeur aqueuse. La théorie biochimique suggère que la photo coagulation du trabéculum entraine une stimulation de certaines réponses biochimiques notamment la modification du métabolisme du collagène. On note une destruction sélective de cellules endothéliales pigmentées, ce qui n'est pas le cas dans l'ALT.

Ce travail porte sur la réponse pressionnelle dans une population mélanoderme.

La population ciblée dans cette investigation était des patients glaucomateux et les sujets hypertones. Les patients qui étaient sous analogues des prostaglandines avaient vu leur traitement relayé par un collyre inhibiteur de l'anhydrase carbonique deux semaines avant le laser comme le suggère certaines études dont celle de Song et al. (2005). Selon Song, les patients sous analogues de prostaglandines n'avaient pas une bonne réduction de la PIO après la SLT. Cette fenêtre thérapeutique aux prostaglandines a été adoptée dans notre cas.

Certains études récentes de Kara notamment (Kara et al., 2001) en Turquie qui a comparé le succès de la SLT chez les patients traités préalablement par prostaglandines et ceux sous combinaison timolol / dorzolamide et n'aurait pas trouvé de différence significative entre les deux groupes.

En effet Kara a pu prouver que $50 \%$ des yeux traités par SLT et qui étaient sous PG avaient une réduction de la $\mathrm{PIO}$ supérieure ou égale à $20 \%$ à un mois après intervention.

La SLT et l'ALT ont une efficacité équivalente comme le démontrent les études de Juzych et martinez de la casa (Juzych et al. 2001; Martinez-delacasa, 2004). Mais si le trabéculum est moins bien visible la SLT avec des 
impacts plus larges permet d'obtenir de meilleurs résultats car moins dépendants de la focalisation des impacts.

Pour Zaninetti (Zaninetti \& Ravinet, 2008) l'énergie variait de 0,7 à 1,3 mJ Dans notre procédure, l'énergie délivrée était comprise entre 0,6 et $0,9 \mathrm{~mJ}$. L'énergie était jugé suffisante dès l'obtention de la bulle en chambre antérieure. Shazly (2012) a aussi retrouvé une énergie similaire au notre : 0,6-0,9 mJ. Seck a retrouvé une énergie variant de 0,5 à $0,9 \mathrm{~mJ}$.

Le nombre d'impact était de 50 sur les $180^{\circ}$ du trabéculum inferieur dans notre cas. Les mêmes paramètres ont été utilisés par Zaninetti, Almeida (Almeida junior et al., 2011) et Kara (Kara et al., 2001) ; tandis que Shazly avait envoyé ses impacts laser soit au niveau du trabéculum inferieur ou nasal. Le nombre d'impacts moyen délivré dans l'étude de Seck était de 71.

Nos patients à la fin de l'intervention avaient reçu une instillation de deux gouttes de collyre hypotonisant dans l'optique de lutter contre les pics d'hypertonie post traitement des premières heures comme rapporté par Melamed et al.

Dans leur série 11\% des yeux traités avaient présenté une élévation de la PIO de plus de $5 \mathrm{~mm}$ hg une heure après SLT (Melamed et al., 2003). Kara et Almeida (Almeida junior et al., 2011; Kara et al., 2001) avait aussi utilisé de la brimonidine collyre en fin d'intervention et avaient respectivement mis leurs patients sous fluorometholone et acetate de prednisolone collyre pour 5 à 7 jours.

Sur ces 130 yeux traités au laser SLT nous notons une moyenne de réduction de la pression intraoculaire de $6,2 \mathrm{~mm}$ hg au premier contrôle et $6,55 \mathrm{~mm}$ hg au second contrôle à 3 mois. Nos valeurs se retrouvent dans la fourchette observée dans la littérature. La moyenne de réduction de la pression après SLT varierait entre 2,09 et $6,5 \mathrm{~mm}$ hg.

Nous avons noté à 1 mois un pourcentage de réduction de la $\mathrm{PIO}$ de 20,2\%. Le pourcentage de réduction de la $\mathrm{PIO}$ par rapport à la pression initiale était de $23,3 \%$ à 6 mois. Il est décrit une réduction de la PIO après la SLT comprise entre 20 et $30 \%$ à court terme (Latina et al.,1996) et une baisse de $30 \%$ de la $\mathrm{PIO}$ à long terme avec un risque d'échappement après un an (Juzych et al., 2004; Weinard \& Althen, 2006). Dans une étude brésilienne Almeida Junior a relevé une réduction de la $\mathrm{PIO}$ de 26,6\% à six mois (Almeida junior et al., 2011).

Nous avons constaté que $50,8 \%$ des yeux traités avaient présenté une réduction de $\mathrm{PIO} \geq 20 \%$ à 1 mois et qu'à 6 mois ils étaient à $52,3 \%$.

Ce résultat à court terme, est superposable aux résultats antérieurement publiés ailleurs. Le pourcentage de succès de la SLT varierait entre 40 et $84 \%$ selon les auteurs (Almeida junior et al., 2011; Realini, 2008). En Egypte, Abdelrahman à un mois post opératoire avait trouvé $69 \%$ (Abdelrahman \& Eltanamly, 2012). 
Nous avons défini comme succès ou bonne réponse pressionnelle une réduction de $\mathrm{PIO} \geq 20 \%$ en nous inspirant d'études antérieures à savoir celle de Kara et al. (2001), qui avait définie le même pourcentage de réduction à un an. Martow et al dans leur étude sur la SLT et le traitement médical adjuvant a aussi défini le succès de la SLT en fixant le pourcentage de réduction de la $\mathrm{PIO} \geq 20 \%$ à 03 et 06 mois (Martow et al., 2011). Aussi Nagar et al dans une étude sur le contrôle de la PIO et sa fluctuation après la SLT a fixé comme succès une réduction de la PIO à 20\% (Nagar et al., 2009). Abdelrahman en Egypte avait défini comme critère de succès de la SLT : une PIO post opératoire $<21 \mathrm{~mm}$ hg et un taux de réduction de la PIO $\geq 20 \%$ (Abdelrahman \& Eltanamly, 2012). Almeida junior quand à lui dans une étude comparative entre SLT et ALT définissait le succès par une pression à 06 mois $\leq 16 \mathrm{~mm}$ hg et une réduction de la $\mathrm{PIO} \geq 25 \%$.

Nous reconnaissons qu'une des limites de notre étude réside dans le temps de suivi court. Néanmoins nous avions pris à profit l'observation de l'équipe de Almeida. En effet elle avait démontré que la grande réduction de la PIO après SLT était observée au $7^{\mathrm{e}}$ jour post opératoire (Almeida junior et al., 2011). Allant dans le même sens, Johnson et al ont montré qu'une diminution de la $\mathrm{PIO}$ à deux semaines post SLT était prédictive d'une réduction pressionnelle à un mois et 3 mois (Johnson et al., 2006).

\section{Conclusion}

Le glaucome est une pathologie fréquence en Afrique. L'un des trois moyens thérapeutiques dans la prise en charge est le traitement physique. La SLT se présente comme un moyen capable de réduire les multi thérapies Bien qu'il ne s'agisse que des résultats à court terme, cette étude prouve que le sujet mélanoderme a une réponse satisfaisante à la SLT. Plus de la moitié des yeux traités ont présenté une réduction pressionnelle de plus de $20 \%$ sur 6 mois. Il faudrait donc une grande série avec un suivi assez long pour le confirmer de façon formelle et aussi évaluer les possibles effets de ce laser au niveau de l'angle iridocornéen.

\section{References:}

1. Abdelrahman, AM. \& Eltanamly, RM. (2012). Selective laser trabeculoplastie in Egyptian patients with primary open angle glaucoma. Middle East Afr J Ophthalmology, 19(3): 299-303.

2. Almeida junior, ED., Pinto, LM., Brandt, RA., \& Prata, TS. (2011). Pattern of intraocular pression reduction following laser trabeculoplasty in open angle glaucoma patients: comparison between selective and nonselective treatment. Clinical Ophthalmology 2011;5:933-6. 
3. Azuara-Blanco, A. \& Burr, J. (2006). The rising cost of glaucoma drugs. Br J Ophthalmol. 2006; 90(2)130-131.

4. Balo, KP., Serouis, AG., Djagnikpo, PA et al. (2006). Que savonsnous de la pression intra oculaire dans la population togolaise. J. Fr Ophtalmol, Jun 29(6) : 629-34.

5. Balo, KP. \& Talabe, M. (1994). Young glaucomatous patients in the Togo population. J Fr Ophtalmol.;17(11): 668-73.

6. Damji, KF., Shah, KC., Rock, WJ et al. (1999) Selective laser trabeculoplasty vs argon laser trabeculoplasty $\mathrm{Br} \mathrm{J}$ Ophthalmol; 83:718-22.

7. Johnson, PB., Katz, LJ., Rhee, DJ. (2006) Selective laser trabeculoplasty: predictive value of early intraocular pressure measurement for success at 3 months. Br J. Ophthalmol; 90:741-3.

8. Juzych, MS., Chopra, V., Banitt, MR et al. (2004). Comparison of long term outcomes of selective laser trabeculoplasty versus argon laser trabeculoplasty in open angle glaucoma. Ophthalmology;111:1853-9.

9. Quigley, HA. (1996). Number of people with glaucoma worldwide. Br J Ophthalmol,80 :389-393.

10. Kara, N., Altan, C., Satana, B et al. (2001). Comparison of selective laser trabeculoplasty success in patients treated with either prostaglandin or timolol/dorzolamide fixed combination. J Ocul. Pharmacol. Ther; 27(4) 339-342.

11. Kramer, TR. \& Noecker, RJ. (2001). Comparison of the morphologic changes after selective laser trabeculoplasty and argon laser trabeculoplasty in human eye bank eyes. Ophthalmology 2001;108:773-9.

12. Latina, MA. \& Park, C. (1995). Selective targeting of trabecular meshwork cells: in vitro studies of pulsed and laser interactions. Exp Eye Res; 60:359-71.

13. Latina, MA., Sibayan, SA., Shin, DH et al. (1998). Qswitched 532 $\mathrm{nm} \mathrm{Nd}$ : YAG laser trabeculoplasty (Selective laser trabeculoplasty): a multi-center pilot clinical study. Ophthalmology;105:2082-8.

14. Latina, MA. \&Tumbocon, JA. (2002). Selective laser trabeculoplasty: a new treatment option for open anfle glaucoma. Curr Oin Ophthalmol; 13 :94-6.

15. Martinez-de-la-casa, JM., Garcia-Feijoo, J., castillo, A et al. (2004). Selective vs argon laser trabeculoplastie: hypotensive efficacy, anterior chamber inflammation and postoperative pain. Eye.18:498502. 
16. Martow, E., Hutnik, CML., Mao, A. (2011). SLT and adjunctive medical therapy a predictive rule analysis. J. Glaucoma; 20(4):26670.

17. Melamed, S., Ben Simon, GJ. \& Levkovitch-verbin, H. (2003). Selective laser trabeculoplasty as primary treatment for open angle glaucoma: a prospective non randomized pilot study. Arch Ophthalmol;121:957-60.

18. Murthy, S. \& Latina, MA. (2009). Pathophysiology of selective laser trabeculoplasty. International Ophthalmology;49(1):89-98.

19. Nagar, M., Luhishi, E., \& Shah, N. (2009). Intraocular pressure control and fluctuation: the effect of treatment with selective laser trabeculoplasty. Br J. Ophthalmol 2009;93:497-501.

20. Pham, H., Mansberger, S. \& Brandt, JD. (2008) Argon laser trabeculoplasty. The gold standard. Survophthal; 53(6) :641-6.

21. Realini, T. (2008). Selective laser trabeculoplasty: A review. J. Glaucoma;17:497-502.

22. Seck, SM., Agboton, G., Dieng, M et al. (2015). La trabéculoplastie au laser selectif: notre experience chez le noir africain. J Fr Ophtalmol; 38: 238-246.

23. Shazly, TA., Latina, MA., Dagianis, JJ., Chitturi, S. (2012). Effect of central corneal thickness on the long-term outcomes of selective laser trabeculoplastie as primary treatment foe ocular hypertension and primary open angle glaucoma. Cornea;0:1-4.

24. Shingleton, BJ., Richter, CU., Dharma, SK et al. (1993). Long term efficacy of argon laser trabeculoplastie. A 10-year follow-up study. Ophthalmology; 100:1324-9.

25. Song J, Lee PP, Epstein DL et al. (2005). High faiilure rate associated with 180 degrees selective laser trabeculoplasty. J. Glaucoma;14:4008.

26. Stein, JD. \& Challa, P. (2007). Mechanism of action and efficacy of argon laser trabeculoplasty and selective laser trabeculoplasty. Curr Opin Ophthalmol 2007;18:140-5.

27. Stein, JD., Kim, DD., Peck, WW., Giannetti, SM., \& Hutton, DW. (2012). Cost-effectiveness of medication compared with laser trabeculoplasty in patients with newly diagnosed open angle glaucoma. Arch Ophthalmol;130(4): 497-505.

28. Tsai, JC. (2006). Medication adherence in glaucoma: approaches for optimizing patient compliance. Curr Opin Ophthalmol. 2006 ; 17(2) : 190-195.

29. Weinard, FS. \& Althen, F. (2006). Long term clinical results of selective laser trabeculoplasty in the treatment of primary open angle glaucoma. Eur J. Ophthalmol;16:100-4. 
30. Wise, JB. \& Witter, SL. (1979). Argon laser therapy for open angle glaucoma. Ach Ophthalmol; 97:319-322.

31. Zaninetti, M. \& Ravinet, E. (2008). Résultats à deux ans de la trabeculoplastie sélective au laser dans le glaucome à angle ouvert et l'hypertonie oculaire. J.Fr.Ophtalmol; 31,10:981-6. 